THE AUTHORS REPLY: The study results referred to by Eisenhut are indeed intriguing, although methodologic limitations such as the nonrandom nature of BCG vaccine uptake complicate interpretation. Findings have been remarkably consistent and are supported by a recent Pediatric Tuberculosis Network European Trials Group collaborative study that showed a $60 \%$ reduction in $M$. tuberculosis infection among children vaccinated with $M$. bovis BCG, as measured by IGRA. ${ }^{1}$ These findings, which imply that BCG vaccination enhances innate immune responses and may affect noncirculating $\mathrm{T}$ cells, challenge developers of tuberculosis vaccines to consider new mechanisms of protection that may precede T-cell stimulation. ${ }^{2}$ We agree that innate immune responses and their potential stimulation by BCG vaccination warrant further study, but the suggestion that a negative IGRA result could serve as a marker of "protection" after documented tuberculosis exposure seems premature. The existing data are not rigorous enough to support its use as a vaccine trial end point. Given the difficulties of quantifying exposure, it would be almost impossible to confirm "protection," and a negative IGRA result would not offer any assessment of acquired immunity, which provides the basis of classic vaccine-induced protection.

Tebruegge et al. emphasize the fact that IGRAs and the tuberculin skin test have similar sensitivity to detect $M$. tuberculosis infection in children and that neither could be regarded as a "superior" test. We trust that this is the message conveyed in our review, which supports World Health Organization recommendations that IGRAs should not replace the tuberculin skin test in low- and middle-income countries. Results from a meta-analysis showed similar accuracy for the diagnosis of tuberculosis infection and disease, with neither test being able to "rule in" or "rule out" the disease. ${ }^{3}$ IGRAs are also more costly and technically complex, with more frequent indeterminate results in young and immunocompromised children. ${ }^{3,4}$ Combined testing in highly vulnerable persons, with either positive test indicating probable infection, improves sensitivity but does not address all limitations. In Table 2 of our review, we indicated the range of sensitivities reported in various studies, but the results are not directly comparable, given the heterogeneity of methods used. We agree that research into better diagnostic tests for childhood tuberculosis remains a top research priority.

Ben J. Marais, M.D., Ph.D.

Sydney Emerging Infectious Diseases and Biosecurity Institute Sydney, NSW, Australia

ben.marais@health.nsw.gov.au

Carlos M. Perez-Velez, M.D.

Grupo Tuberculosis Valle-Colorado

Medellín, Colombia

Since publication of their article, the authors report no further potential conflict of interest.

1. Basu Roy R, Sotgiu G, Altet-Gómez N, et al. Identifying predictors of interferon- $\gamma$ release assay results in pediatric latent tuberculosis: a protective role of bacillus Calmette-Guerin? A pTB-NET collaborative study. Am J Respir Crit Care Med 2012;186:378-84. 2. Lalvani A, Bakir M, Millington KA, Dosanjh D, Soysal A. BCG and protection against Mycobacterium tuberculosis infection. Lancet 2006;367:391-2.

3. Mandalakas AM, Detjen AK, Hesseling AC, Benedetti A, Menzies D. Interferon-gamma release assays and childhood tuberculosis: systematic review and meta-analysis. Int J Tuberc Lung Dis 2011;15:1018-32.

4. Connell TG, Zar HJ, Nicol MP. Advances in the diagnosis of pulmonary tuberculosis in HIV-infected and HIV-uninfected children. J Infect Dis 2011;204:Suppl 4:S1151-S1158.

DOI: 10.1056/NEJMc1210173

\title{
More on Failures of Cobalamin Assays in Pernicious Anemia
}

TO THE EDITOR: Carmel and Agrawal (July 26 issue $)^{1}$ stress that current assays for the measurement of serum cobalamin (vitamin $\mathrm{B}_{12}$ ) may produce falsely elevated results in patients harboring autoantibodies against intrinsic factor, whereas this was not the case for older assays of cobalamin. The authors suggest the implementation of control samples containing such antibodies. This step will illuminate the problem but will not ensure correct measurement of cobalamin in individual patient samples.

Polyethylene glycol precipitation was recently suggested as a possible means of removing the antibodies. ${ }^{2}$ We repeated the procedure used in this study with five normal serum samples and found that polyethylene glycol precipitates a large part of the transcobalamin (69 to 74\%) and thereby an important amount of cobalamin. We conclude that precipitation with polyethylene glycol would lead to an incorrect measurement of cobalamin in all samples tested. The formerly used method of pretreating samples by boiling at an acidic $\mathrm{pH}$ was efficient but is not feasible in typical laboratories today. 
Measurement of the active vitamin $\mathrm{B}_{12}$, or holotranscobalamin, instead of total cobalamin, ${ }^{3}$ would circumvent the problem. Another solution would be to demand that producers of commercial diagnostic kits exchange hog intrinsic factor with a binding protein not recognized by autoantibodies against intrinsic factor.

Eva Greibe, M.Sci.

Johan Arendt, B.Med.Sci.

Ebba Nexo, M.D., Dr.Med.Sci.

Aarhus University Hospital

Aarhus, Denmark

e.nexo@dadlnet.dk

No potential conflict of interest relevant to this letter was reported.

1. Carmel R, Agrawal YP. Failures of cobalamin assays in pernicious anemia. N Engl J Med 2012;367:385-6. [Erratum, N Engl J Med 2012;367:976.]

2. Yang DT, Cook RJ. Spurious elevations of vitamin $B_{12}$ with pernicious anemia. N Engl J Med 2012;366:1742-3.

3. Nexo E, Hoffmann-Lucke E. Holotranscobalamin, a marker of vitamin B-12 status: analytical aspects and clinical utility. Am J Clin Nutr 2011;94:359S-365S.

DOI: $10.1056 /$ NEJMc1210169

TO THE EDITOR: Carmel and Agrawal recently added 8 cases to the 11 previously reported in which the presence of intrinsic factor autoantibodies was associated with misleadingly normal cobalamin values. However, they incorrectly suggest that this phenomenon also explains my previous finding that neither normal levels of cobalamin nor normal levels of the cobalamin-dependent metabolite, methylmalonic acid (MMA), preclude the presence of cobalamin-responsive clinical disorders. ${ }^{1,2}$ In fact, when the data in my previous study were reanalyzed to include only those patients in whom intrinsic factor autoantibodies were not detected, $67 \%$ of the 39 patients with one low cobalamin level had a normal second value, $53 \%$ of the 74 patients with two normal cobalamin values had high MMA levels, and 75\% of the 29 patients with a clinical response had consistently normal cobalamin values (with both cobalamin values $>300$ pg per milliliter in $61 \%$ ), and MMA values were less than $376 \mathrm{nmol}$ per liter in $54 \%$ of the 29 patients with a clinical response (unpublished data). In conclusion, "false" normal cobalamin values often result from intraindividual variation, and clinical responses to cobalamin therapy occur despite normal cobalamin and MMA values. Thus, there is still no "gold standard" for the diagnosis of cobalamin deficiency, and therapeutic trials are warranted when the clinical picture is consistent with this disorder. ${ }^{3}$
Lawrence R. Solomon, M.D.

Yale University School of Medicine

New Haven, CT

lawrence.solomon@yale.edu

No potential conflict of interest relevant to this letter was reported.

1. Solomon LR. Cobalamin-responsive disorders in the ambulatory care setting: unreliability of cobalamin, methylmalonic acid, and homocysteine testing. Blood 2005;105:978-85.

2. Solomon LR. Tests for cobalamin-responsive disorders are unreliable. Blood 2005;106:1137-8.

3. Green R. Unreliability of current assays to detect cobalamin deficiency: "nothing gold can stay." Blood 2005;105:910-1. DOI: 10.1056/NEJMc1210169

TO THE EDITOR: Carmel and Agrawal highlight the problems of using the results of the serum competitive-binding luminescence assay (CBLA) in diagnosing pernicious anemia (also previously reported by $\mathrm{me}^{1}$ ) but fail to suggest the option of testing serum levels of holotranscobalamin ${ }^{2}$ to detect cobalamin deficiency. This assay circumvents the problem of interference from intrinsic factor autoantibodies that characterizes the CBLA. In addition, the level of intrinsic factor autoantibodies that is most likely to cause interference has not been reported. Unless every CBLA is also assessed for intrinsic factor autoantibodies, and the level of serum cobalamin is assessed in relation to the level of intrinsic factor autoantibodies, the CBLA may have little meaning. The practicality and cost of such an approach are prohibitive, particularly in a high-volume, high-throughput routine diagnostic laboratory. The use of a widely and routinely used assay that may give a false negative result is particularly worrying. There are arguments for ${ }^{3}$ and against ${ }^{4}$ the use of holotranscobalamin, but the time is ripe to consider it as an alternative to cobalamin to screen for and diagnose cobalamin deficiency in a routine diagnostic laboratory setting.

\section{Vinod Devalia, M.B., Ch.B.}

Princess of Wales Hospital

Bridgend, United Kingdom

devaliacymru@btinternet.com

No potential conflict of interest relevant to this letter was reported.

1. Devalia V. Diagnosing vitamin B-12 deficiency on the basis of serum B-12 assay. BMJ 2006;333:385-6.

2. Heil SG, de Jonge R, de Rotte MC, et al. Screening for metabolic vitamin B12 deficiency by holotranscobalamin in patients suspected of vitamin B12 deficiency: a multicentre study. Ann Clin Biochem 2012;49:184-9.

3. Nexo E, Hoffmann-Lücke E. Holotranscobalamin, a marker of vitamin B-12 status: analytical aspects and clinical utility. Am J Clin Nutr 2011;94:359S-365S.

4. Carmel R. Holotranscobalamin: not ready for prime time. Clin Chem 2012;58:643-5.

DOI: 10.1056/NEJMc1210169 
THE AUTHORS REPLY: The letters from Greibe and Nexo and from Devalia offer various suggestions to address the failures we reported in the ability of competitive-binding luminescence assay (CBLA) to identify low serum cobalamin levels. Greibe and Nexo misunderstand the value of monitoring CBLA modification and performance with antibody-positive serum, emphasize issues related to the use of polyethylene glycol that are tangential to CBLA malfunction in pernicious anemia, and suggest exploration of alternative cobalamin-binding proteins without warning that some proteins introduce analogue-related artifacts.

More important, the recommendation by Devalia and by Greibe and Nexo to replace testing for cobalamin with testing for holotranscobalamin is highly controversial and premature, because too little is known about what factors influence holotranscobalamin levels. ${ }^{1}$ Detailed, clinically relevant studies are scarce, and those which do exist are often less positive in their recommendations on its use $\mathrm{e}^{2}$ than the clinically uninformative biochemical surveys of subclinical deficiency, which show marginal advantages for the use of holotranscobalamin. ${ }^{1}$ It deserves emphasis that specificity, not sensitivity, is the diagnostic weakness of cobalamin-related testing in clinical practice: all markers, including cobalamin, have sensitivities of more than $95 \%$ (barring assay error) for clinical deficiency but have poor specificities. ${ }^{1}$ Indeed, the specificity of holotranscobalamin may be poorer than that of cobalamin $(56 \% \text { vs. } 66 \%)^{2}$; still more troubling is the fact that its confounders remain largely unexplored. For example, the half-life of holotranscobalamin, measured in minutes, may be oversensitive to fluxes between the gut and the bloodstream. ${ }^{1}$ Studies are needed to determine whether common influences too transient to affect cobalamin stores or status (e.g., a few weeks of poor cobalamin intake or drug-induced malabsorption) yield levels of holotranscobalamin that are misleadingly low.

When deficiency is clinically obvious, a single biochemical abnormality usually suffices diagnostically, barring assay errors. ${ }^{1,3}$ Should the cobalamin assay, which is the first-line option for many reasons, ${ }^{1}$ remain error-prone, the holotranscobalamin assay would be a poor substitute until the questions about holotranscobalamin interpretation are properly addressed. Methylmalonic acid, which was used to "validate" holotranscobalamin, seems preferable despite its imperfections. ${ }^{1}$ Additional metabolic tests can help when the findings are equivocal or the patient has subclinical deficiency. ${ }^{1}$ (Testing for intrinsic factor antibody has value in the diagnosis of pernicious anemia ${ }^{3}$ independent of CBLA error-related considerations.)

Solomon's letter, which mischaracterizes our data (we identified 9 new cases, not 8, and our letter [and the table in the related Supplementary Appendix] summarized the results of 7 cases reported individually by others, not 11), is unconvincing despite the unpublished reanalyses of his earlier paper. Using two definitions of normal serum cobalamin, the paper reported that $86 \%$ of 37 patients with symptomatic deficiency had cobalamin levels above 200 pg per milliliter and 54\% had levels above 300 pg per milliliter. ${ }^{4}$ Those unprecedented and to this day unreproduced frequencies of failure encouraged the mischaracterization of cobalamin as being insensitive in patients with clinically expressed deficiency. As detailed in a critique of the study, ${ }^{5}$ only assay errors (the Centaur CBLA, then known to fail in $73 \%$ of deficient serum specimens, ${ }^{5}$ was used, and the cobalamin levels reported in the study frequently oscillated spontaneously), misdiagnoses, or both can explain Solomon's $86 \%$ and $54 \%$ rates of false normal cobalamin levels. ${ }^{4}$ False normal rates are much smaller in clinically expressed deficiency, ${ }^{1,3}$ except when assays fail. As for Solomon's closing sentence, we agree with it completely. ${ }^{3}$

Ralph Carmel, M.D.

New York Methodist Hospital

Brooklyn, NY

rac9001@nyp.org

Yash Pal Agrawal, M.B., B.S., Ph.D.

Weill Cornell Medical College

New York, NY

Since publication of their letter, the authors report no further potential conflict of interest.

1. Carmel R. Biomarkers of cobalamin (vitamin B-12) status in the epidemiologic setting: a critical overview of context, applications, and performance characteristics of cobalamin, methylmalonic acid, and holotranscobalamin II. Am J Clin Nutr 2011; 94:348S-358s.

2. Schrempf W, Eulitz M, Neumeister V, et al. Utility of measuring vitamin B12 and its active fraction, holotranscobalamin, in neurological vitamin B12 deficiency syndromes. J Neurol 2011; 258:393-401.

3. Carmel R. How I treat cobalamin (vitamin B12) deficiency. Blood 2008;112:2214-21.

4. Solomon LR. Cobalamin-responsive disorders in the ambulatory care setting: unreliability of cobalamin, methylmalonic acid, and homocysteine testing. Blood 2005;105:978-85.

5. Carmel R. Is testing for clinical cobalamin deficiency truly unreliable? Blood 2005;106:1136-7.

DOI: $10.1056 / N E J M c 1210169$ 